\title{
Les principales méthodes d'évaluation des efforts provoqués par la houle sur divers types d'ouvrages
}

PAR

\author{
Y. Allouard
}

Franlab

J.-L. Chenot

Institut Français du Pétrole
ET
Nous avons distingué deux types de structures correspondant à des méthodes d'approche différentes:

- Les structures de grande dimension soumises à l'action d'une houle périodique de faible ou moyenne amplitude,

- les structures comportant des éléments pour lesquels il n'est plus possible de négliger les forces de traînée.

Dans le premier cas, nous développons une théorie en fluide parfait irrotationnel, adaptée au calcul par éléments finis ou par fonctions spline dans le cas linéaire ou non linéaire. Dans le second cas, nous résolvons les équations de la dynamique pour la structure soumise à un ensemble de forces, la force de trainée étant évaluée à l'aide d'une approximation de type Morison.

\section{Modèles \\ en fluide parfait irrotationnel}

\section{1 - THEORIE LINEAIRE}

a) Rappel des équations [1]

Les fluide étant supposé parfait et irrotationnel nous pouvons résoudre le problème en $\phi$ potentiel des vitesses. Les diverses notations sont précisées sur la figure 1 :

- le plan $x$ Oy est la surface libre au repos $S$

- $F$ est le fond supposé horizontal situé à la profondeur $h$

- Cest la surface mouillée de la structure

- D est le domaine fluide compris entre $S$, $F$ et $C$

- A est le demi créte à creux de la houle incidente dont la di- rection de propagation fait un angle $\nu+\pi$ avec l'axe $O x$, et $T$ sa période, $\omega=2 \pi / T$.

Posons en outre :

$\eta$ hauteur de la surface libre,

$\vec{n}$ normale à $(C)$ au point considéré,

$\overrightarrow{v_{c}}$ vitesse du point considéré de $(C)$

$P$ pression dans le fluide

$\rho$ masse volumique de l'eau

Alors les équations du problème sont

$$
\begin{array}{ll}
\Delta \Phi=0 & \text { dans }(D) \\
\frac{\partial \Phi}{\partial z}=0 & \text { sur }(F)
\end{array}
$$
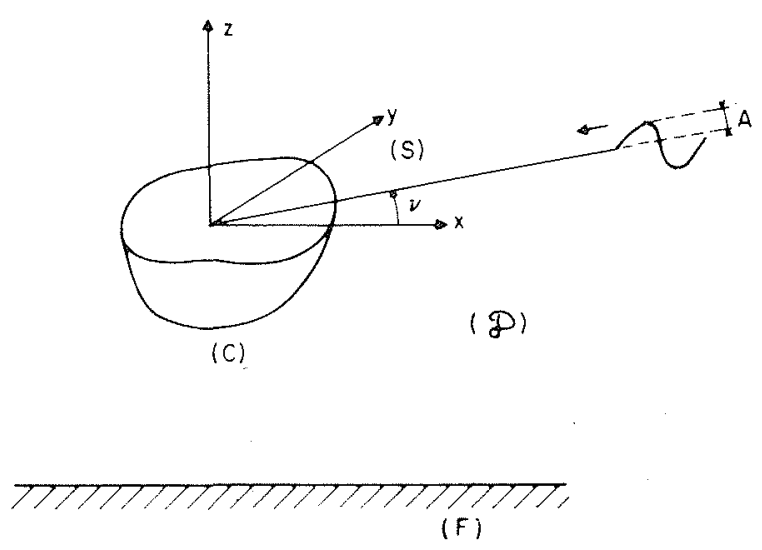

11 


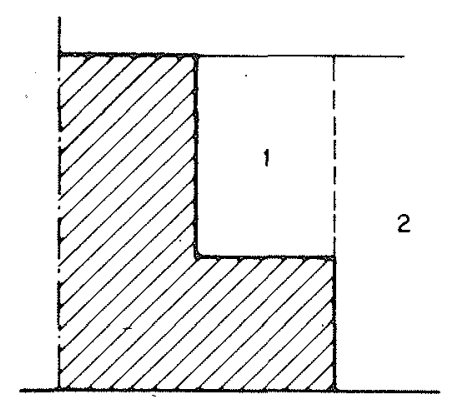

21

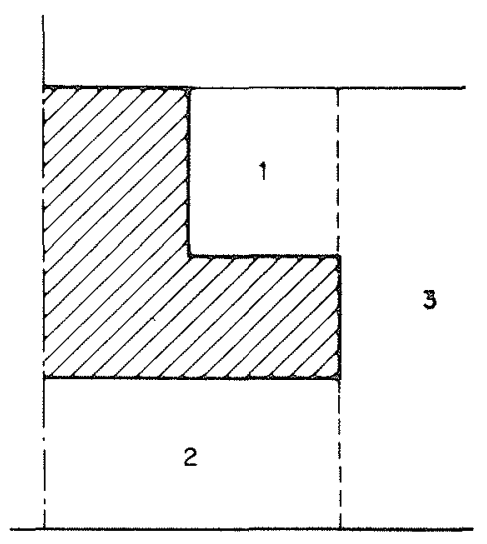

3।

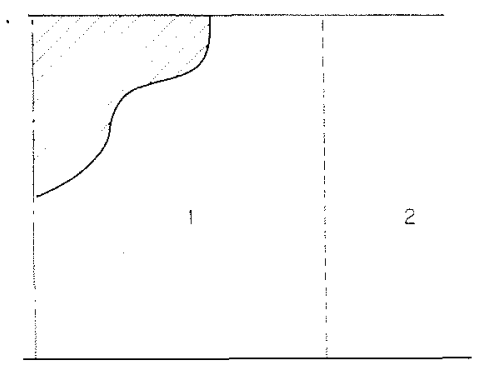

41

$$
\begin{aligned}
& \frac{\partial \Phi}{\partial n}=\vec{v}_{c} \cdot \vec{n} \quad \text { sur } \quad(C) \\
& \frac{\partial \eta}{\partial t}=\frac{\partial \Phi}{\partial z} \\
& \frac{\partial \Phi}{\partial t}+g \cdot \eta=0 \\
& P=-\rho \frac{\partial \Phi}{\partial t} \quad \operatorname{dans}(D)
\end{aligned}
$$

Il est commode de décomposer $\Phi$ sous la forme

$$
\Phi=\Phi^{I}+\Phi^{D}+\Phi^{R}
$$

avec la signification : I pour incident

$$
\begin{aligned}
& D \text { pour diffracté } \\
& R \text { pour radiation }
\end{aligned}
$$

On sait que la hauteur de la surface libre de la houle incidente peut s'écrire:

$$
\eta^{I^{\prime}}=A \operatorname{Cos}\left[k_{1}(x \cos \nu+y \sin \nu)+\omega t\right]
$$

En fait, il est commode de considérer que toutes les fonctions sont complexes, nous utiliserons done de préférence :

$$
\eta^{\prime}=A e^{i\left|k_{1}(x \cos \nu+y \sin \nu)+\omega t\right|}
$$

où $k_{1}$ vérifie $k_{1}$ th $k_{1} h=\omega^{2} / g$

On peut alors vérifier que le potentiel incident complexe s'écrit :

$$
\Phi^{I}=a \operatorname{ch} k_{1}(z+h) e^{\left.i \mid k_{1}(x \cos v+y \sin \nu)+\omega t\right)}
$$

avec

$$
a=\frac{i g \mathrm{~T}}{2 \pi \operatorname{ch} k_{1} h}
$$

En théorie linéaire, les solutions sont écrites sous la forme

$$
\Phi(x, y, z, t)=\phi(x, y, z) e^{i \omega t}
$$

Ce qui permet d'écrire la condition de surface libre

$$
\begin{aligned}
\frac{\partial \Phi}{\partial z}-\sigma \phi & =0 \quad \operatorname{sur}(S) \\
\sigma & =\frac{\omega^{2}}{g}
\end{aligned}
$$

D'autre part. le mouvement de ia structure est décrit par lo vecteur vitesse du centre de gravité dont les composants sont

$$
\left(V_{x} e^{i \omega t}, V_{y} e^{i \omega t}, V_{z} e^{i \omega t}\right)
$$

et par le vecteur rotation instantance:

$$
\left(\Omega_{a} e^{i \omega t}, \Omega_{\beta} e^{i \omega t}, \Omega_{\gamma} e^{i \omega t}\right)
$$

Nous posons alors :

$\phi^{R}=V_{x} \phi_{x}+V_{y} \phi_{y}+V_{z} \phi_{z}+\Omega_{\alpha} \dot{\phi}_{\alpha}+\Omega_{\beta} \phi_{\beta}+\Omega_{\gamma} \phi_{\gamma}$

Appelons $\phi_{i}$ l'un des potentiels $\phi_{x}, \ldots, \phi_{\gamma} \phi^{D}$. Pour que $\phi$ de (6) vérifie les équations (1) à (4), il suffit que:

$$
\begin{array}{lll}
\Delta \phi_{i}=0 & \text { dans } & (D) \\
\frac{\partial \phi_{i}}{\partial z}=0 & \text { sur } & (F) \\
\frac{\partial \phi_{i}}{\partial z}-\sigma \phi_{i}=0 & \text { sur } & (S)
\end{array}
$$

et (3) est remplacée sur $(C)$ par:

$$
\begin{aligned}
& \frac{\partial \phi^{D}}{\partial n}+\frac{\partial \phi^{l}}{\partial n}=0 \\
& \frac{\partial \phi_{x}}{\partial n}=\vec{i} \cdot \vec{n} \\
& \frac{\partial \phi_{y}}{\partial n}=\vec{j} \cdot \vec{n} \\
& \frac{\partial \phi_{z}}{\partial n}=\vec{k} \cdot \vec{n} \\
& \frac{\partial \phi_{\alpha}}{\partial n}=(\overrightarrow{G M} \wedge \vec{n}) \cdot \vec{i} \\
& \frac{\partial \phi_{\beta}}{\partial n}=(\overrightarrow{G M} \wedge \vec{n}) \cdot \vec{j} \\
& \frac{\partial \phi_{\gamma}}{\partial n}=(\overrightarrow{G M} \wedge \vec{n}) \cdot \vec{k}
\end{aligned}
$$

Enfin, pour que le problème potentiel soit entièrement déterminé il faut préciser le comportement à l'infini des solutions soit :

$$
\left|\phi_{i}\right|^{2} \sim \frac{C_{i}}{\sqrt{x^{2}+y^{2}}} \text { pour } x^{2}+y^{2} \rightarrow \infty
$$


Introduisons maintenant les déplacements du centre de gravité par rapport à sa position d'équilibre et les déplacements angulaires de la structure soit :

$$
\xi=\left[\begin{array}{l}
x \\
y \\
z \\
\alpha \\
\beta \\
\gamma
\end{array}\right] e^{i \omega t}
$$

Alors $\frac{d \xi}{d t}=i \omega \xi$ et $\frac{d^{2} \xi}{d t^{2}}=--\omega^{2} \xi$

Soit $t^{A}$ le torseur des forces d'Archimède dues au mouvement, après linćarisation, on peut écrire:

$$
t^{A}=A \xi
$$

où $A$ est une matrice 6.6. Soit $m$ la masse de la structure et $T$ son tenseur d'inertie, $I_{3}$ la matrice 3,3 unité, posons

$$
M=\left[\begin{array}{cc}
m I_{3} & 0 \\
0 & T
\end{array}\right]
$$

La résultante des forces hydrodynamiques sur la structure est, d'après (5) et (6)

$$
\vec{f}=-\rho i \omega \iint_{C}\left(\phi^{l}+\phi^{D}+\phi^{R}\right) \vec{n} d S e^{i \omega r}
$$

et de mème, le moment résultant est

$$
\vec{\mu}=-\rho i \omega \iint_{C}\left(\phi^{l}+\phi^{D}+\phi^{R}\right)(G \vec{M} \wedge \vec{n}) d S e^{i \omega t}\left(13^{\prime}\right)
$$

Par conséquent, d'après (10), (12), (13) et (13'), le torseur des forces hydrodynamiques peut s'écrire:

$$
\tau^{H}=\tau^{I}+\tau^{D}+\omega^{2} B \xi
$$

(où $B$ est une matrice d'ordre 6 complexe).

Finalement, il est clair que les équations de la dynamique pour la structure, peuvent se mettre sous la forme du systeme linéaire

$$
\left[-\omega^{2}(M+B)+A\right] \xi=\tau^{I}+\tau^{D}
$$

\section{b) Structure de révolution :}

Dans le cas où la structure est de révolution, et bien que le problème ne soit pas entièrement de révolution, l'introduction des coordonnées cylindriques $r, \theta, z$ permet de se ramener à l'étude d'un certain nombre de problèmes bidimensionnels.

Chaque potentiel est développé en série de Fourrier du type :

$$
\phi(x, y, z)=\sum_{m=0}^{\infty} \phi_{m}(r, z) \cos m \theta
$$

(les termes en $\sin m \theta$ disparaissent du fait que le problème possède un plan de symétrie). Le laplacien nul s'écrit :

$$
\frac{\partial^{2} \phi_{m}}{\partial r^{2}}+\frac{1}{r} \frac{\partial \phi_{m}}{\partial r}+\frac{\partial^{2} \phi_{m}}{\partial z^{2}}-\frac{m^{2}}{r^{2}} \phi_{m}=0
$$

Les conditions aux frontières s'écrivent en fonction de $r$ et $z$ uniquement, enfin, dans le plan $(r, z)$ il apparaît une condi- tion aux limites supplémentaire pour $r=0$; on montre facilement que :

$$
\begin{aligned}
\frac{\partial \phi_{0}}{\partial r}=0 & (r=0) \\
\phi_{m}=0 & (r=0, m \neq 0)
\end{aligned}
$$

L'on cherche à résoudre le problème en séparant les variables, la condition à l'infini conduit à choisir la série :

$$
\begin{aligned}
\phi=\sum_{m=0}^{\infty}\left[a_{m 1} f_{1}(z)(\right. & \left.J_{m}\left(k_{1} r\right)-i Y_{m}\left(k_{1} r\right)\right) \\
& \left.+\sum_{n=2}^{\infty} a_{m n} f_{n}(z) K_{m}\left(k_{n} r\right)\right] \cos m \theta
\end{aligned}
$$

\section{$J$, $Y$ et $K$ sont les fonctions de Bessel}

$$
\begin{aligned}
& f_{1}(z)=\frac{\sqrt{2} \operatorname{ch}\left(k_{1}(z+h)\right)}{\left[h+\operatorname{sh}^{2}\left(k_{\mathrm{j}} h\right) / \sigma\right]^{1 / 2}} \\
& f_{n}(z)=\frac{\sqrt{2} \cos \left(k_{n}(z+h)\right)}{\left[h-\sin ^{2}\left(k_{n} h\right) / \sigma\right]^{1 / 2}}
\end{aligned}
$$

$k_{n}$ solution $n^{\circ}(n-1)$ de l'équation $k \operatorname{tgg} k h=\frac{\omega^{2}}{g}$

\section{c) Modèles pour les structures de révolution.}

L'une des méthodes la plus simple, consiste à essayer de satisfaire la condition (3) sur (C) à l'aide d'un développement du type (17).

En fait, cela n'est pas possible avec un nombre raisonnable de termes dans le développement en série, sauf dans le cas du cylindre vertical. Dans le cas où la structure peut être schématisée par deux cylindres concentriques (ensemble pile-caisson), il est possible de séparer le plan $(r, z)$ en zones distinctes dans lesquelles une solution du type (17) est convenable. Il suffit ensuite d'assurer le raccordement des potentiels et des dérivées normales aux frontières entre ces zones.

Cette méthode a été utilisée pour les modèles TOUR avec deux zones (voir fig. 2) et MOUVE avec trois zones (voir fig. 3).

Dans le cas ou la méridienne de la structure de révolution est quelconque, il est apparu plus efficace de choisir une zone fluide finie entourant la structure dans laquelle on construit une solution du problème, et une zone extéricure infinie où la solution est du type (17) (voir fig. 4), la continuité du potentiel et de sa dérivée normale étant assurée à la frontière. Nous avons comparé deux méthodes discrètes

la première utilisant une base de fonctions spline (voir [4], modèle TOURQ) la seconde utilisant la méthode des éléments finis avec des triangles à trois points (voir [2] : modèle COREV).

Nous avons pu constater que la précision des deux modèles était tout à fait comparable, et que le second modèle est beaucoup plus économique.

\section{d) Modele pour une structure quelconque}

La seule hypothèse que nous ayons faite sur la structure, est qu'elle forme un ensemble parfaitement rigide. Le volume fluide est encore divisé en deux zones (voir fig. 5). 


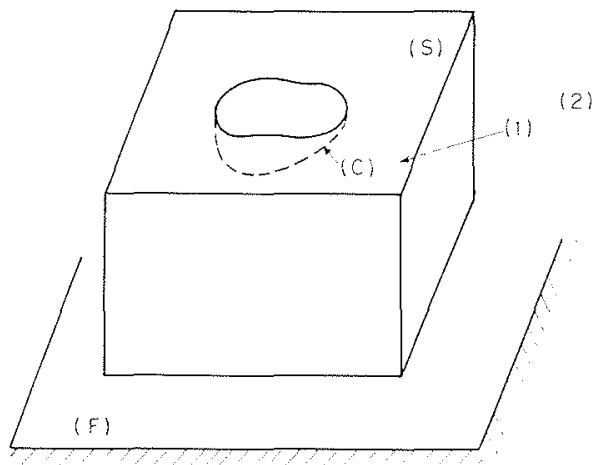

5 /

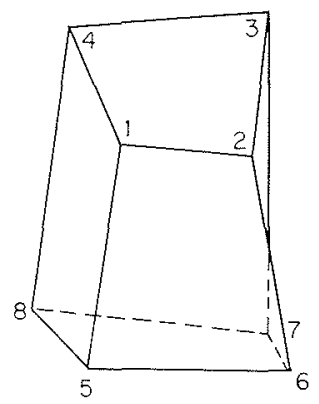

$6 /$

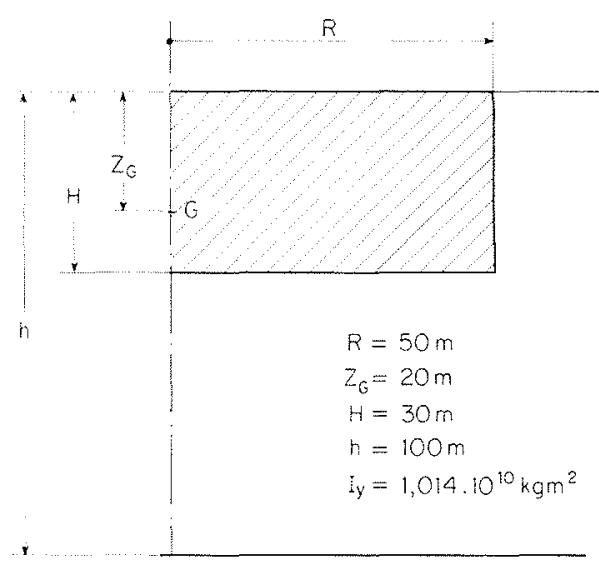

71
Dans la première zone, on utilise des éléments finis à huit nœuds (voir fig. 6), dans la seconde zone, un développement analy tique du type (17) ne suffit plus, il faut utiliser la forme générale

$\phi=\sum_{m=0}^{\infty}\left[\left(a_{m !} \cos m \theta+b_{m 1} \sin m \theta\right) f_{1}(z)\left(J_{m}\left(\left(k_{1} r\right)-i Y_{m}\left(k_{1} r\right)\right)\right.\right.$

$\left.+\sum_{n=2}^{\infty}\left(a_{m n} \cos m \theta+b_{m n} \sin m \theta\right) f_{n}(z) K_{m}\left(k_{n} r\right)\right]$

Le calcul complet est réalisé par le programme TRITON.

\section{e) Exemple numérique}

Pour illustrer ce qui précède, nous avons étudié le mouvement d'un cylindre flottant (fig. 7) par le programme COREV avec un maillage très fin et pour différentes périodes de houle.

Le méme calcul a été exécuté par le programme TRITON pour trois périodes de houle avec un maillage esquissé sur la figure 8. L'ensemble des résultats est donné par les figures 9 à 11. Ajoutons que dans ce cas précis, le temps d'exécution des calculs est de :

\section{COREV $10 \mathrm{~s}$ \\ TRITON $40 \mathrm{~s}$}

sur l'ordinateur CDC 7600.

\section{2 - THEORIE NON LINEAIRE}

Les modèles précédents peuvent être généralisés dans le cadre de la théorie qui suit. Nous utiliserons un développement en série de perturbation, en nous limitant au second ordre, la théorie sera développée uniquement dans le cas d'une structure fixe.

a) Développement au second ordre (voir [3] p. 653 et suivantes).

Rappelons tout d'abord les équations générales.

$\mathrm{Si}$$$
z=\eta(x, y, t)
$$

est l'équation de la surface libre, nous aurons les deux conditions

$$
\begin{aligned}
& \frac{\partial \Phi}{\partial z}=\frac{\partial \eta}{\partial t}+\frac{\partial \Phi}{\partial x} \frac{\partial \eta}{\partial x}+\frac{\partial \Phi}{\partial y} \frac{\partial \eta}{\partial y} \\
& \frac{\partial \Phi}{\partial t}+\frac{1}{2}(\operatorname{grad} \Phi)^{2}+g \eta=0
\end{aligned}
$$

valable pour $z=\eta$

Le principe de la méthode consiste à chercher une solution de la forme

$$
\begin{aligned}
& \eta_{1}=\epsilon \eta_{1}+\epsilon^{2} \eta_{2}+\cdots \\
& \Phi=\epsilon \Phi_{1}+\epsilon^{2} \Phi_{2}+\cdots
\end{aligned}
$$

et à ramener les termes de surface libre au niveau moyen $z=0$ par le développement.

$$
\Phi(x, y, z, t)=\Phi(x, y, 0, t)+\eta \frac{\partial \Phi}{\partial z}(x, y, 0, t)+\cdots
$$

On reporte (20) et (21) dans (18) et (19) en utilisant (22). Si on identifie les termes en $\epsilon$ on obtient les équations :

$$
\begin{aligned}
& -\frac{\partial \eta_{1}}{\partial t}+\frac{\partial \Phi_{1}}{\partial z}=0 \\
& \text { pour } z=0 \\
& \frac{\partial \Phi_{1}}{\partial t}+g \eta_{1}=0
\end{aligned}
$$

tandis que les termes en $\epsilon^{2}$ conduisent à

$$
\begin{gathered}
-\frac{\partial \eta_{2}}{\partial t}-\frac{\partial \Phi_{1}}{\partial x} \frac{\partial \eta_{1}}{\partial x}-\frac{\partial \Phi_{1}}{\partial y} \frac{\partial \eta_{1}}{\partial y}+\eta_{1} \frac{\partial^{2} \Phi_{1}}{\partial z^{2}}+\frac{\partial \Phi_{2}}{\partial z}=0 \\
\text { pour } z=0 \\
\frac{\partial \Phi_{2}}{\partial t}+g \eta_{2}+\eta_{1} \frac{\partial^{2} \Phi_{1}}{\partial z \partial t}+\frac{1}{2}\left(\operatorname{grad} \Phi_{1}\right)^{2}=0 \\
\text { pour } z=0
\end{gathered}
$$

Nous poserons maintenant

$$
\begin{aligned}
& \epsilon \Phi_{1}=\phi_{1} e^{i \omega t} \\
& \epsilon^{2} \Phi_{2}=\phi_{2} e^{2 i \omega t}
\end{aligned}
$$



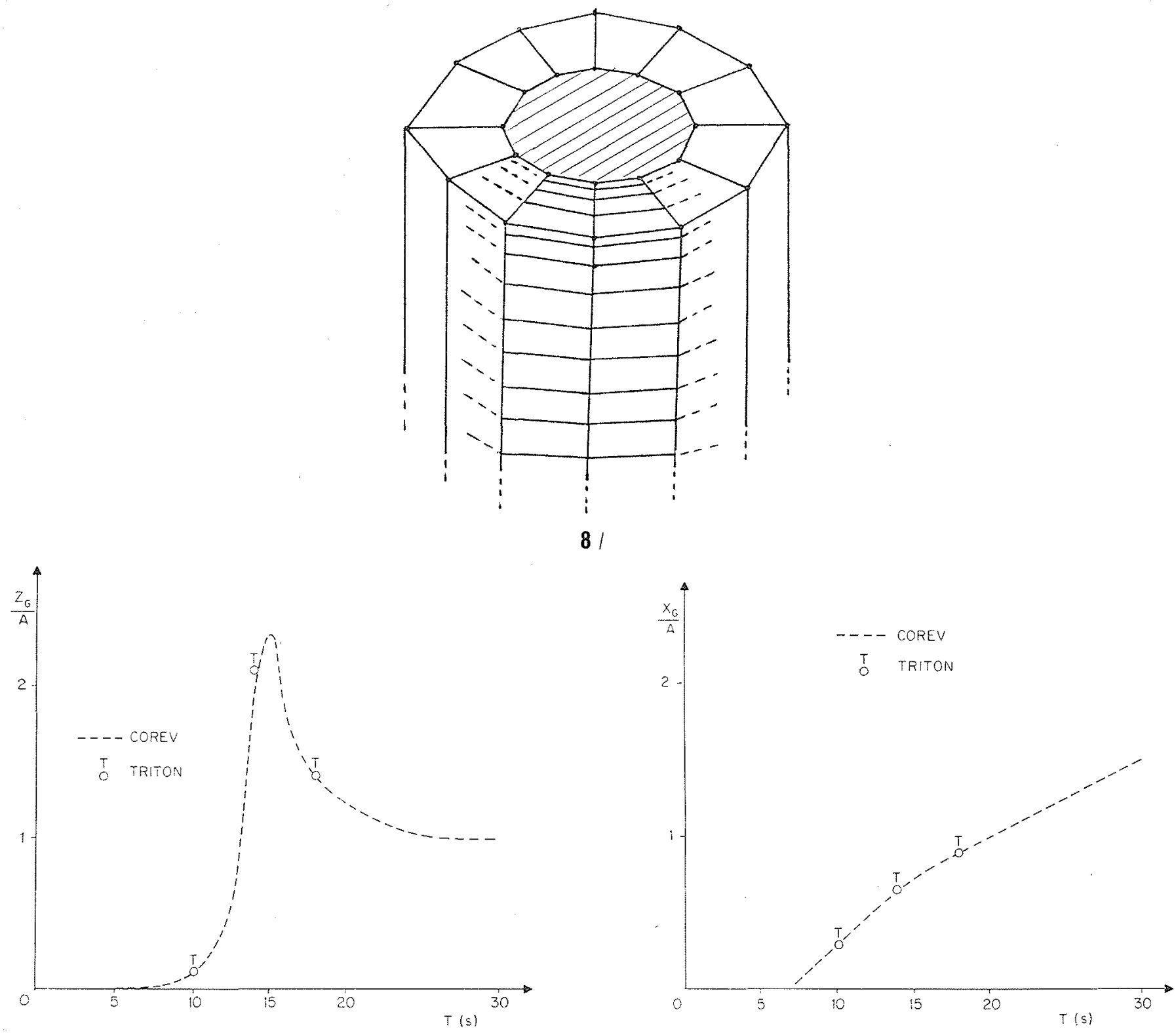

9 /

111

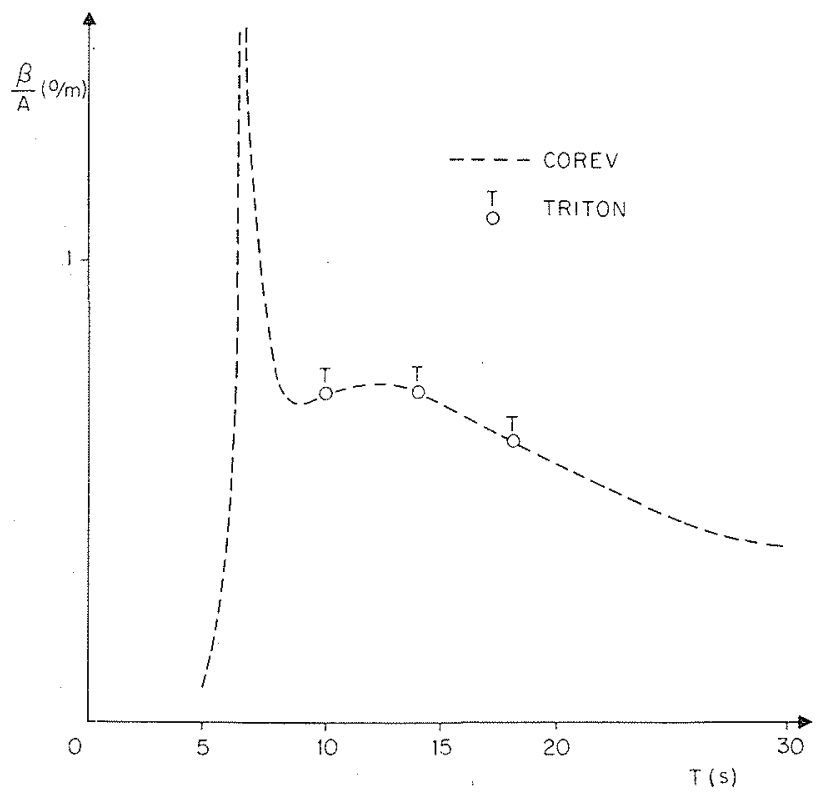


alors (23) et (24) s'écrivent après élimination de $\eta_{1}$

$$
\frac{\partial \phi_{1}}{\partial z}-\frac{\omega^{2}}{g} \phi_{1}=0 \quad(z=0)
$$

Cette équation est identique à celle obtenue pour le cas linéaire, ce qui montre que l'ordre 1 peut être résolu de façon indépendante.

D'autre part (25) et (26), après élimination de $\eta_{2}$, conduisent à l'équation

$$
\begin{aligned}
\frac{\partial \phi_{2}}{\partial z}-\frac{4 \omega^{2}}{g} \phi_{2}=-\frac{i}{4 \omega}\left[2 \left(\frac{\partial \phi_{1}^{2}}{\partial x}+\frac{\partial \phi_{1}^{2}}{\partial y}\right.\right. & +\frac{3 \omega^{4}}{g} \phi_{1}^{2} \\
& \left.\left.-\phi_{1} \frac{\partial^{2} \phi_{1}}{\partial z^{2}}\right)\right]
\end{aligned}
$$

Le premier membre de (28) est formellement identique à celui de (27) (il suffit de remplacer $\omega$ par $1 / 2 \omega$ ), le second membre de (28) ne contient que des termes en $\Phi_{1}$ que nous pouvons supposer déjà calculés.

Les non-linéarités pour un corps immobile, ne se manifestent explicitement qu'à la surface libre. En ce qui concerne les conditions sur $(C)$ le potentiel incident étant connu (houle de Stokes du second ordre), il faut écrire:

$$
\frac{\partial \phi_{1}^{D}}{\partial n}=-\frac{\partial \phi_{1}^{I}}{\partial n} \quad \frac{\partial \phi_{2}^{D}}{\partial n}=-\frac{\partial \phi_{2}^{I}}{\partial n} .
$$

Pour la condition à l'infini, nous avons introduit une approximation supplémentaire :

$\phi_{1}^{D}$ est raccordé à un développement asymptotique du type (17) ou $\left(17^{\prime}\right)$.

$\phi_{2}^{D}$ est raccordé à un développement du méme type dans lequel

$$
\begin{aligned}
& k_{1} \text { est solution de } k \text { th } k h=\frac{4 \omega^{2}}{g} \\
& k_{n} \text { est solution de } k \operatorname{tg} k h=-\frac{4 \omega^{2}}{g}
\end{aligned}
$$

ce qui équivaut à négliger certains des termes issus du second membre de (28) au-delà de la zone discrètisée.

\section{b) Modèles non linéaires :}

Les programmes pour les structures de révolution à méridienne quelconque, ont pu être étendus au cas non linéaire en donnant naissance aux modèles TOURQN et COREVN qui sont actuellement en cours de test. Pour le cas tridimensionnel, le modèle TRITON2 est en cours d'élaboration.

\section{Intégration des équations du mouvement d'une structure en régime transitoire}

\section{1 - EQUATIONS GENERALES}

Les équations de la dynamique s'écrivent pour le corps solide

$$
\begin{aligned}
m \Gamma_{G} & =\sum_{i} F_{i} \\
\frac{d}{d t}(T \Omega) & =\sum_{i} M_{i}
\end{aligned}
$$

$m$ est la masse du solide.

$\Gamma_{G}$ est l'accélération du centre de gravité de la structure,

$T$ est le tenseur d'inertie de la structure,

$\Omega$ est le vecteur rotation instantané,

$F_{i}$ et $M_{i}$ sont les forces et moments extérieurs.

Les forces extérieures sont :

- le poids de la structure

- la pousséc d'Archimede qui dépend du volume immergé

- les forces de diffraction et de radiation qui peuvent etro déduites de la théorie précédente

- la force de trainée qui peut etre approximée par une formule du type Morizon, soit :

$$
F=\frac{1}{2} \rho C_{d i} \iint_{S_{i}}|\vec{U}-\vec{v}|\left(\vec{U}_{r}-\vec{v}_{r}\right) d S
$$

où $C_{d}$ est le coefficient de trainée.

$\vec{U}$ est la vitesse du fluide.

$\vec{v}$ la vitesse du point considéré de la structure.

$S_{i}^{\prime}$ designe la partie de la surface immergé pour laquelle

$$
(\vec{U}-\vec{\imath}) \cdot \vec{n}<0
$$

$\vec{n}$ étant la normale extérieure au corps, enfin l'indice $r$ désigne la composante nomale pour $\vec{U}$ et $\vec{v}$

\section{2 - PRINCIPE DU MODELE}

La surface de la structure est discrétisée en facettes, ce qui permet d'évaluer les forces de trainée par intégration numérique ainsi que le volume immergé, lorsque l'on connait la position du centre de gravité de la structure $X_{G}$, sa vitesse $V_{G}$, les trois angles de roulis, tangage, lacet $A$ et le vecteur rotation instantanée $\Omega$. D'autre part, (30) s'écrit :

$$
T \frac{d \Omega}{d t}=-\frac{d T}{d t} \Omega+\Sigma M_{i}
$$

et il est facile de vérifier que cette dernière équation peut elle même s'écrire :

$$
\ddot{A}=g(t, X, \dot{X}, A, \dot{A})
$$

si l'on reformule (29) sous la forme équivalente

$$
\ddot{X}=f(t, X, \dot{X}, A, \dot{A})
$$

on constate que (31) et (32) constituent un systeme non linéaire du second ordre de 6 équations différentielles qu'il est possible d'intéger pas à pas dans le temps à l'aide d'un des nombreux sous-programmes classiques adaptés à ce problème. 


\section{Conclusion}

Ces deux types de méthodes possèdent chacune leurs limitations:

la thérorie en fluide parfait irrotationnel ne permet pas de tenir compte des forces de trainée et se trouve donc inapplicable pour les structures en forme de treillis,

- la seconde méthode permet de simuler le comportement d'un fluide réel, mais ne permet d'étudier que les phénomenes transitoires pour les systemes faiblement amortis où le régime permanent ne peut étre atteint qu'après une trop longue integration dans le temps.
En fait, l'expérience prouve que la plupart des cas réels peuvent être traités avec l'un ou l'autre de ces modèles.

\section{Bibliographie}

[1] WEHAUSEN J.V. - Annual Rey. of Fluid Mech., 1971, 38010.

[2] ZIENKIEWICZ O.C. - La méthode des éléments finis appliquee a l'art de l'ingenieur, 1973, Ediscience (Paris).

[3] Encyclopedia of Physics. - Volume IX, Fluid Dynamics III, 1960 Springer Verlag (Berlin)

[4] LAURENT P.J. - Approximation et optimisation, 1972, Herman.

\section{Discussion}

Président : M. P. WILLM

M. le Président remercie M. Chenor pour son exposé très clair qui résume fort bien une question très compliquée et ouvre la discussion.

M. RÉmÉviéras rappelle que la "Société Energie thermique des Mers " avait essayé en vraie grandeur, notamment au large de Brest (pointe Saint-Mathieu), des flotteurs « anti-houle " destinés à la pose de la conduite sous-marine d'amenée d'eau froide pour l'usine qu'elle projetait d'implanter à Abidjan ('). Ces flotteurs étaient constitués par un cylindre creux vertical d'une vingtaine de mètres de hauteur, assemblé à sa partie inférieure à un long flotteur cylindrique horizontal immergé à une distance suffisante de la surface pour que les mouvements engendrés par la houle soient négligeables. Les oscillations - et en particulier le pilonnement de tels flotteurs sous l'action de la houle avaient une grande importance pratique pour la pose de la conduite sous-marine par des fonds allant à plus de $500 \mathrm{~m}$. Les méthodes de calcul exposées par M. CHENOT seraient-elles applicables à de tels flotteurs antihoule?

Cela dépend des dimensions du flotteur et en particulier du cylindre vertical qu'il comporte, répond $\mathrm{M}$. CHENOT. Si les forces de traînée ne peuvent être négligées, on peut toujours calculer les masses ajoutées et les moments d'inertie d'un tel système en supposant le fluide irrotationnel, et ensuite utiliser un autre programme - dont je n'ai pas parlé ici parce que d'un point de vue théorique il est moins fondé - qui rajoute les forces de traînée et utilise une intégration numérique pour le phénomène transitoire.

Les calculs exposés, demande M. SAGNer (Sté Bertin) ont-ils fait l'objet de confrontations avec des essais sur modèle ou sur structures réelles? De tels essais sont-ils envisagés?

Le premier modèle de révolution que je vous ai présenté, répond M. CHENOT, a été testé sur des essais en bassin. Les résultats ont été satisfaisants, ce qui nous a encouragés à continuer dans cette voie.

Pour le modèle tri-dimensionnel, il n'y a que des tests vis-à-vis d'autres modèles; il n'est pas sûr que ledit modèle soit correct pour un corps de forme tout à fait quelconque. On n'a pas encore fait de tests par rapport à des "essais en bassin " en raison de problèmes un peu terre à terre de maillage... Ainsi, pour une plate-forme à plusieurs pieds, par exemple, il faut donner un grand nombre de nœuds; or, entrer à la main les trois coordonnées d'environ 6000 nouds, puis un nombre équivalent de données décrivant les huit sommets de chaque élément, cela représente un travail monstrueux...

(1) Voir " Compte Rendu des IVe Journées de l'Hydraulique : les Energies de la mer ", Question VIII, pp. 685-725 (S.H.F., 1956).
On a mis au point un programme de maillage automatique qui permet de ne donner qu'un certain nombre de sections du corps étudié. Mais, actuellement, ce programme n'est pas encore tout à fait opérationnel; il n'est applicable qu'à des corps assez simples comme ceux que j'ai montrés, des corps ne comportant qu'un seul élément. Lorsqu'il y a plusieurs pieds, le programme ne fonctionne pas.

Or, les résultats que nous avons concernent surtout des platesformes; donc actuellement, on n'a pas encore pu faire de tests directs; mais ce sera fait dès que possible.

Quelle est l'importance relative des forces de trainée (dues à la viscosité) du corps et des termes non-linéaires calculés en fluide parfait? demande M. SABATON (L.N.H.).

Les programmes qui ont été écrits, précise M. CHEnor, ne s'appliquent qu'à des corps fixes. Le programme tri-dimensionnel qui doit fonctionner pour un corps flottant n'est pas encore au point; il n'est donc pas possible de répondre actuellement à la question posée par M. SABATON.

Pourriez-vous dessiner quelques formes de structures que vous pouvez calculer, demande M. BEAUdesSON (G.E.M.-Hersent).

En s'aidant de quelques schémas, M. CHENOT indique, comme suit, le champ d'application des méthodes qu'il a exposées.

Dans les problèmes bi-dimensionnels, on est limité par la capacité de l'ordinateur mais en fait, on peut traiter le cas de corps très compliqués.

Par contre, actuellement, en tri-dimensionnel on est beaucoup plus limité; de façon opérationnelle on peut traiter essentiellement des corps qui ne comportent qu'une seule partie, tel un parallélépipède rectangle; on pourra aussi " modéliser » un bateau.

Il serait souhaitable de pouvoir dans un plan horizontal prendre en compte plusieurs sections afin de modéliser des plates-formes pétrolières à 3,4 ou 5 pieds, des catamarans, etc. Ceci est pratiquement au point; le programme fonctionne; on pourrait " rentrer » ces données à la main, mais cela représente quinze jours à trois semaines de travail. Pour que ce calcul soit effectivement possible, il faut que le "maillage automatique " soit parfaitement opérationnel; en outre, ce maillage automatique éliminerait beaucoup de sources d'erreurs car la "sortie » de ce maillage doit être compatible avec le programme Triton.

Pour les corps de révolution, les seules limitations sont les hypothèses de dérive. Il faut qu'il n'y ait pas de parties trop fines (pas de cylindre supérieur de diamètre trop faible dans le cas évoqué plus haut) car alors les forces de traînée seraient non négligeables; mais on peut encore apporter à ces résultats une correction en utilisant des formules de type Morrison pour tenir 
compte éventuellement des forces de traînée. Un autre programme permet alors d'intégrer les équations de la dynamique, compte tenu d'un certain nombre de forces agissant sur le corps

Mais je dois préciser que le mouvement moyen du corps doit être nul; on ne peut pas étudier le cas d'un corps qui se déplace à vitesse constante (on n'a pas de programme pour le calcul de la résistance "de vague").

M. SAGNER (Sté Bertin) pose la question suivante :

Vous avez fait allusion, dans vos réponses et dans votre résumé, à l'adjonction de forces de traînée. Que pensez-vous de la validité de cette adjonction? $\left({ }^{2}\right)$

Il se pose, à ce sujet, plusieurs problèmes, précise M. CHENor Tout d'abord, si l'on fait une intégration numérique avec des conditions un peu sévères, il peut se trouver que le mouvement du corps ne soit plus un «petit mouvement». En ce cas, les masses ajoutées qu'on a calculées ne sont plus valables puisqu'elles sont

(2) Le fait de disposer d'un calcul très précis des forces d'inertie ne permet-il pas de déduire des résultats expérimentaux des valeurs correctes des forces de trainée? calculées pour de "petits mouvements. C'est là un point important à souligner.

D'autre part, pour le calcul des forces de traînée proprement dites, on utilise des formules de type Morrison car on ne connaît rien de mieux actuellement. Les résultats dépendent énormément des conditions particulières au problème. Dans certains cas, c'est assez bon. Dans d'autres, il convient d'être très réservé.

Il est vrai que les formules de Morrison ont été testées par de très nombreuses personnes; d'ailleurs elles ont été déterminées à partir de l'expérience.

Mais le programme n'a fait l'objet que de peu de tests car on dispose de peu de résultats du fait que plusieurs phénomènes entrent en ligne de compte et qu'il est malaisé de séparer l'action des forces de traînée.

Si aux forces d'inertie s'ajoutent des forces de traînée, l'expérience permet de trouver la somme des deux. Mais on ne peut pas dire que les forces d'inertie sont correctes et les forces de trainée également; il se pourrait qu'il y ait un effet de compensation entre les deux composantes. Tout ce qu'on peut dire, c'est que globalement le programme donne des résultats satisfaisants.

M. le Président clôt la discussion et donne la parole à M. SABATON pour l'exposé de sa communication. 